\title{
Effect of airway opening manoeuvres on thoraco-abdominal asynchrony in anaesthetized children
}

\author{
A. Reber*, S.A. Bobbià*, J. Hammer", F.J. Frei*
}

Effect of airway opening manoeuvres on thoraco-abdominal asynchrony in anaesthetized children. A. Reber, S.A. Bobbià, J. Hammer, F.J. Frei. (C) ERS Journals Ltd 2001.

ABSTRACT: Thoraco-abdominal asynchrony is frequently encountered during inhalation anaesthesia in children with adenotonsillar hypertrophy causing an upper airway obstruction. The study goal was to evaluate the impact of different airway opening manoeuvres on thoraco-abdominal asynchrony as a measure of airway obstruction.

Thirty anaesthetized children (aged 2-8 yrs; sevoflurane 3\% in 50\% oxygen/nitrous oxide) were studied prior to elective adenotonsillectomy using respiratory inductance plethysmography to record ribcage and abdominal wave forms as a basis for calculation of the phase angle. Five airway situations were compared: 1) baseline (unsupported mandible); 2) chin lift; 3) chin lift combined with continuous positive airway pressure of $10 \mathrm{cmH}_{2} \mathrm{O}$; 4) jaw thrust; and 5) jaw thrust combined with continuous positive airway pressure of $10 \mathrm{cmH}_{2} \mathrm{O}$.

Three children had complete upper airway obstruction at baseline and were excluded from the study. With chin lift, thoraco-abdominal asynchrony improved in three patients, worsened in three patients and was unchanged in 21 patients. Additional continuous positive airway pressure during chin lift did not markedly reduce thoracoabdominal asynchrony (phase angle $89 \pm 43^{\circ}, p=0.33$ ). Jaw thrust resulted in a significant decrease of the phase angle (from $106 \pm 53^{\circ}$ at baseline to $65 \pm 49^{\circ}, \mathrm{p}<0.01$ ); when combined with continuous positive airway pressure, no further effect on thoracoabdominal asynchrony was found $\left(72 \pm 44^{\circ}\right)$.

In anaesthetized children with adenotonsillar hypertrophy, airway opening manoeuvres have distinct effects on thoraco-abdominal asynchrony. Delivery of continuous positive airway pressure and jaw thrust can be the first airway opening manoeuvres to improve breathing patterns. Chin lift without additional continuous positive airway pressure should be used with caution in these patients because it may convert partial into almost complete airway obstruction.

Eur Respir J 2001; 17: 1239-1243.
Depts of *Anaesthesia and "Paediatrics, University Children's Hospital of Basel, Basel, Switzerland.

Correspondence: A. Reber

Dept of Anaesthesia

University of Basel/Kantonsspital

CH-4031 Basel

Switzerland

Fax: 41612657320

Keywords: Adenotonsillar hypertrophy respiratory inductance plethysmography

thoraco-abdominal asynchrony

upper airway obstruction

Received: May 262000

Accepted after revision February 24 2001

This study was supported by a grant of the Swiss National Science Foundation (3200-056034.98).
Adenotonsillectomy is one of the most common paediatric operations [1]. Close communication between all medical staff involved in the care of these children is fundamental to their well-being [2]. The anaesthetic management of all these children is centred around the preservation and control of the upper airway. Upper airway obstruction (UAO) can easily occur because inhalation anaesthetics decrease pharyngeal muscle tone [3]. Hypertrophy of the adenoids and the tonsils can further worsen upper airway patency, which is one of the main reasons for UAO in children [4]. In an animal model, an increase in UAO correlates with increasing thoraco-abdominal asynchrony (TAA) [5]; UAO is measured and quantified using respiratory inductance plethysmography to record ribcage $(\mathrm{RC})$ and abdominal $(\mathrm{AB})$ wave forms with subsequent calculation of the phase angle [6]. TAA decreases in children with resolving UAO due to laryngotracheobronchitis [7]. Asynchronous or paradoxical breathing patterns can occur during general anaesthesia with volatile anaesthetic agents
[3]. The paradoxical pattern is most pronounced when UAO becomes progressively worse [8]. When this occurs, delivery of continuous positive airway pressure (CPAP) may be the first in a series of attempted airway opening manoeuvres such as chin lift and jaw thrust to reduce the obstruction [8]. The purpose of the present study was to determine if these interventions in anaesthetized children decrease TAA.

\section{Material and methods}

This study examined consecutively, 30 children (aged 2-8 yrs) who were scheduled for adenotonsillectomy. Children with cranio-facial dysmorphism, deformities of the chest or spine and children with neuromuscular disorders were excluded from the study. The protocol was approved by the Ethics Committee of the University Children's Hospital, Basel. Prior to a patient's participation in the study, the child's parents gave written informed consent. 


\section{Anaesthesia}

The patients were rectally premedicated with $0.3 \mathrm{mg} \cdot \mathrm{kg}^{-1}$ midazolam, $15 \mathrm{~min}$ before anaesthesia. All patients were monitored with pulse oximetry, electrocardiography, and blood pressure measurement using a Datex cardiocap II monitor (Helsinki, Finland). Inhalation anaesthesia was induced with sevoflurane (in steps of $1.5 \%$ volume per 3 breaths up to $8 \%$ volume) in a circle-system anaesthesia machine. The steady state of sevoflurane concentration was at $3 \%$ volume with $50 \%$ oxygen/nitrous oxide [9].

\section{Airway manoeuvres}

The head position was standardized, the head being slightly extended with an angle of $110^{\circ}$ between the horizontal plane of the operating table and the line connecting the lateral corner of the eye and the tragus of the ear. This angle leads to maximally widened oral and nasal upper airways [10]. Each manoeuvre was standardized and performed for $1 \mathrm{~min}$ by the same investigator to eliminate inter-investigator variability in all children. For each child the following manoeuvres were determined. After induction of anaesthesia, baseline measurement was performed with each patient's chin unsupported (face mask adapted). Thereafter, chin lift was performed single handed without protrusion of the mandible. The molars were lightly contacted, the lips remained open. Thus, complete mouth closure was not achieved. For the third situation, in addition to chin lifting, CPAP of $10 \mathrm{cmH}_{2} \mathrm{O}$ was applied in the circle system in order to dilate or splint the upper airway. From then on, jaw thrust was performed bimanually with the jaw being displaced upwards and anteriorly (Esmarch manoeuvre), which enabled the mouth to remain completely open. This manoeuvre with a maximal mandibular protrusion was performed at zero endexpiratory airway pressure followed by CPAP of $10 \mathrm{cmH}_{2} \mathrm{O}$. At the end of this procedure, each patient's trachea was intubated and anaesthesia was maintained for the subsequent elective surgery.

The study was stopped if a child's upper airway was totally obstructed (no breathing sounds detected by the investigator). If airway manoeuvres failed to establish an open airway, artificial respiration with neuromuscular blockade and consecutive endotracheal intubation was performed.

\section{Measurement of thoraco-abdominal asynchrony}

$\mathrm{RC}$ and $\mathrm{AB}$ motions were depicted from the output of an AC coupled respiratory inductance plethysmograph (RIP; Respitrace $\mathbb{R}$, Non-invasive Monitoring Systems NIMS, Miami Beach, FL, USA) operating in the alternating current mode. The exact position of the bands placed at the levels of the nipples and upper abdomen was carefully examined during the investigations [11]. The operator was blinded to the recordings. All data were analysed with a $50 \mathrm{~Hz}$ resolution of signals using specifically designed software
(RespiEvents 4.2e, Non-invasive Monitoring Systems NIMS).

The phase angle was used to measure TAA [6]. According to the method of Agostoni and MognONI [12], phase angle is calculated using an $\mathrm{x}-\mathrm{y}$ plot of $\mathrm{RC}$ versus $\mathrm{AB}$ motion display ("Lissajous figure", KonnoMead diagram) in a sine wave dependent formula: sine phase angle $=\mathrm{m} \cdot \mathrm{s}^{-1}$. Where " $\mathrm{m}$ " describes the distance between the intercepts of the RC/AB loop on a line drawn parallel to the $\mathrm{x}$-axis, which is placed at half the distance between the maximal and minimal $\mathrm{RC}$ excursions; and "s" is the maximal AB excursion. Values for the phase angle fall between $0^{\circ}$ for complete synchrony and $180^{\circ}$ for complete asynchrony (paradoxical breathing). The loops were identified as an indicator of relative timing between $\mathrm{RC}$ and $\mathrm{AB}$ motions during the respiratory cycle.

In addition, the following parameters were calculated. 1) The total time spent in asynchrony (TTA), meaning the percentage of time during one breath in which the RC and $\mathrm{AB}$ motion signals were in opposite directions ( $\mathrm{RC}$ inward and $\mathrm{AB}$ motion outward or vice versa). 2) The lag of $\mathrm{RC}$ relative to $\mathrm{AB}$ motion at start of inspiration and expiration, respectively [13]. These thoraco-abdominal coordination indices are sine wave independent values (in contrast to the phase angle) between 0 and $100 \%$, where $0 \%$ indicates perfect synchrony and $100 \%$ complete asynchrony (the phase angle $=180^{\circ}$ corresponding to $100 \%$ asynchrony). 3) The duty cycle or ratio of inspiration to total time $\left(t \mathrm{t} / t_{\text {tot }}\right)[14]$.

\section{Data analyses and statistics}

Variables and statistical data were calculated from the mean and SD of 20 consecutive respiratory cycles in the steady state [14]. The five airway situations were compared by analysis of variance for repeated measures. For post hoc comparisons, Tukey's test was applied if appropriate and probability values were calculated. Spearman rank correlation coefficient was applied to analyse possible relationships between variables. A p-value $<0.05$ was considered to be significant.

\section{Results}

Thirty children (aged 2-8 yrs) were studied (4.4 \pm $1.6 \mathrm{yrs}$, mean $\pm \mathrm{SD})$. Body weight ranged from 9$24 \mathrm{~kg}(18 \pm 4 \mathrm{~kg})$ and height ranged from $78-125 \mathrm{~cm}$ $(100 \pm 12 \mathrm{~cm})$. Their case histories revealed nocturnal apnoea in six patients. From clinical inspection, three children were excluded from the study protocol because of a complete UAO with the chin unsupported (two patients) and the occurrence of a central apnoea at chin lift (one patient). Except for these three patients, pulse oximetry values remained $\geqslant 97 \%$ throughout the study protocol. No significant change of respiratory frequency or heart rate occurred. The mean data of the 27 patients are summarized in table 1 . There was no correlation between TAA and age in this study population. 
Table 1. - Impact of airway opening manoeuvres

\begin{tabular}{|c|c|c|c|c|c|}
\hline & Chin unsupported & Chin lift & Chin + CPAP & Jaw thrust & $\mathrm{Jaw}+\mathrm{CPAP}$ \\
\hline Phase angle ${ }^{\circ}$ & $106 \pm 53$ & $106 \pm 54$ & $89 \pm 42$ & $65 \pm 49 * \#$ & $72 \pm 44^{* \#}$ \\
\hline IAI \% & $70 \pm 29$ & $60 \pm 29$ & $55 \pm 26$ & $41 \pm 26^{* \# \oplus}$ & $46 \pm 26^{* \# \oplus}$ \\
\hline EAI \% & $39 \pm 23$ & $35 \pm 20$ & $30 \pm 13$ & $28 \pm 19 *$ & $28 \pm 21 *$ \\
\hline TTA $\%$ & $49 \pm 24$ & $46 \pm 22$ & $40 \pm 15$ & $33 \pm 20$ *\# & $32 \pm 14$ *\# \\
\hline$t \mathrm{I} / t$ tot & $0.43 \pm 0.10$ & $0.43 \pm 0.09$ & $0.38 \pm 0.04 * \#$ & $0.39 \pm 0.05$ & $0.38 \pm 0.05^{* \#}$ \\
\hline $\mathrm{RF} \min ^{-1}$ & $35 \pm 8$ & $35 \pm 8$ & $37 \pm 10$ & $36 \pm 9$ & $37 \pm 10$ \\
\hline HR $\min ^{-1}$ & $105 \pm 14$ & $104 \pm 15$ & $101 \pm 15$ & $105 \pm 15$ & $103 \pm 16$ \\
\hline
\end{tabular}

Data are presented as mean $\$$ SD. CPAP: continuous positive airway pressure; IAI: inspiratory asynchrony index; EAI: expiratory asynchrony index; TTA: total time spent in asynchrony; $t \mathrm{I} / t$ tot: ratio of inspiration to total time; RF: respiratory frequency; HR: heart rate. *: signigicantly different from "chin unsupported" $(\mathrm{p}<0.05)$; : significantly different from chin lift; ${ }^{\uparrow}$ : significantly different from chin lift + CPAP.

At chin lift, TAA measured by phase angle improved in three patients, worsened in three patients and was unchanged in 21 patients. Additional CPAP during chin lift did not markedly reduce TAA (phase angle $89 \pm 43^{\circ}, \mathrm{p}=0.33$ ). Data for phase angle, asynchrony indices and $t \mathrm{I} / t$ tot are presented in table 1 and figures $1-3$. Jaw thrust resulted in a significant decrease of the phase angle (from $106 \pm 53^{\circ}$ at baseline to $\left.65 \pm 49^{\circ}, \mathrm{p}<0.01\right)$, although in combination with CPAP no further effect on TAA (phase angle $72 \pm 44^{\circ}$ ) was found. Similar statistical significances were found for the asynchrony indices and the duty cycle, thus favouring jaw thrust with and without CPAP (table 1, figs. 1-3).

\section{Discussion}

The major finding was that in children with adenotonsillar hypertrophy, moderate and severe TAA motions were commonly seen during sevoflurane anaesthesia. In these patients, airway opening manoeuvres had distinct effects on TAA. Delivery of CPAP and jaw thrust can be the first airway opening manoeuvres to improve breathing patterns. Chin lift

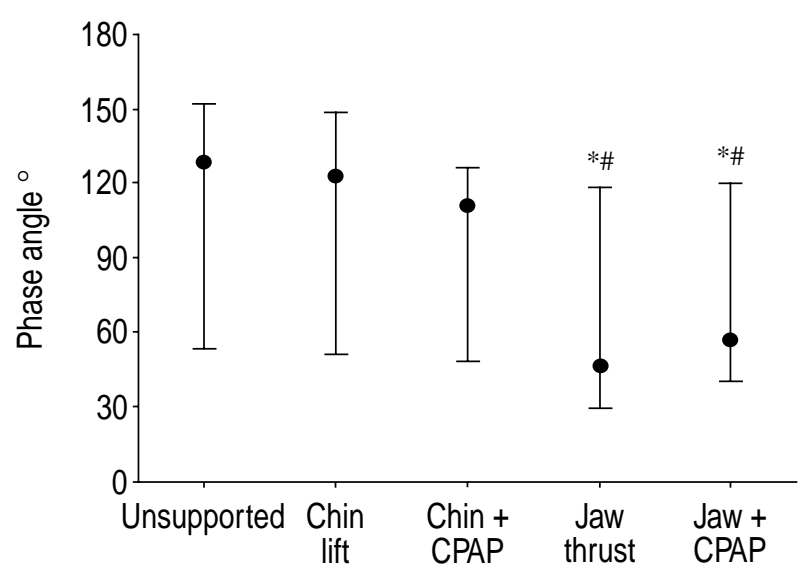

Fig. 1. - The effect of chin lift and jaw thrust with and without continuous positive airway pressure (CPAP) on phase angle. Phase angle data were skewed and all data are therefore presented uniformly as median and $25-75 \%$ interquartile range. ${ }^{*}$ : significantly different from "chin unsupported" $(\mathrm{p}<0.05)$; ${ }^{\#}$ : significantly different from chin lift $(\mathrm{p}<0.05)$. Jaw thrust with and without CPAP significantly improved thoraco-abdominal asynchrony. without additional CPAP should be used with caution in these patients, because it may convert partial into almost complete airway obstruction.

During general anaesthesia, a large scatter of phase angles occurred. At baseline, the majority of patients had moderate and severe asynchronous breathing patterns that may correlate with clinical observations of UAO $[15,16]$. In the present study, five patients
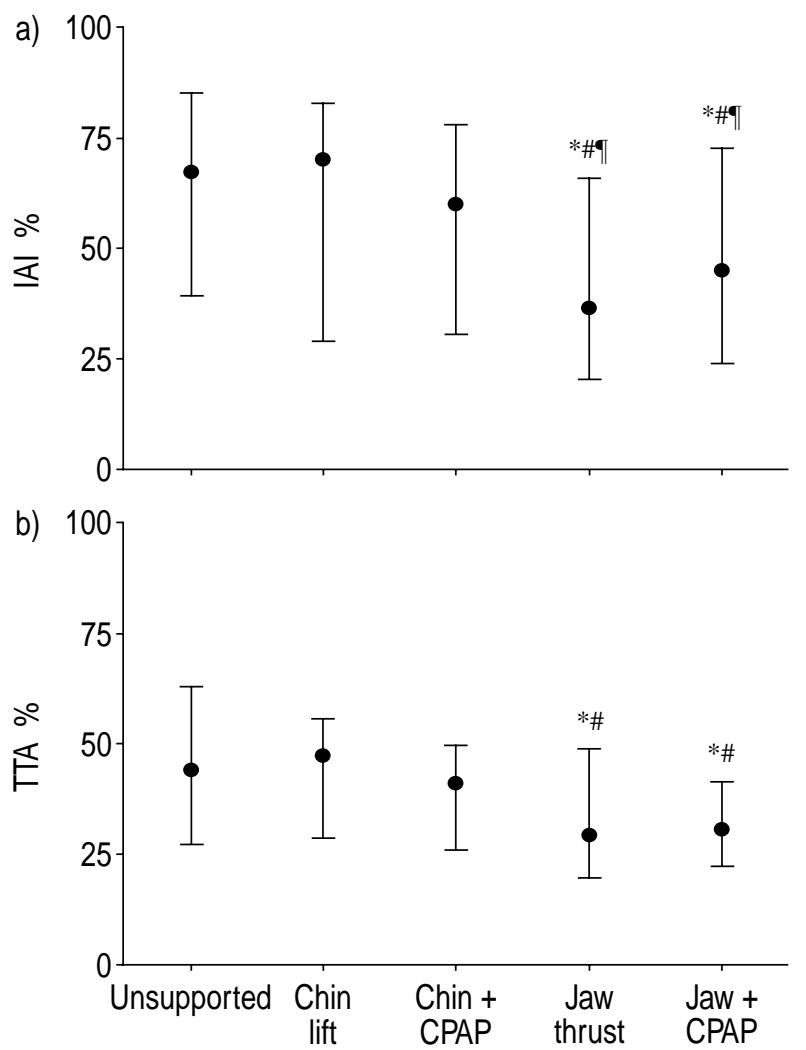

Fig. 2. - The effect of chin lift and jaw thrust with and without continuous positive airway pressure (CPAP) on: a) the lag of ribcage relative to abdominal motion at start of inspiration (inspiratory asynchrony index; IAI) and on b) total time spent in asynchrony (TTA). Because phase angle data (fig. 1) were skewed, all data are presented uniformly as median and $25-75 \%$ interquartile range. *: significantly different from "chin unsupported" $(\mathrm{p}<0.05) ;{ }^{\#}$ : significantly different from chin lift $(\mathrm{p}<0.05) ;{ }^{\uparrow}$ : significantly different from chin lift + CPAP $(p<0.05)$. Jaw thrust with and without CPAP significantly improved thoraco-abdominal asynchrony. 


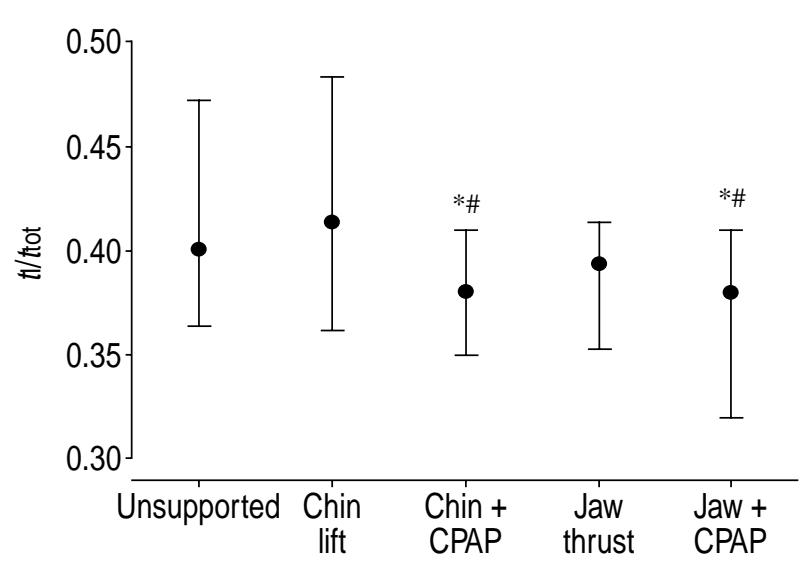

Fig. 3. - The effect of chin lift and jaw thrust with and without continuous positive airway pressure (CPAP) on the ratio of inspiration to total time ( $t \mathrm{I} / t_{\text {tot }}$ ). Because phase angle data (fig. 1) were skewed, $t \mathrm{I} / t$ tot data are presented uniformly as median and $25-75 \%$ interquartile range. *: significantly different from "chin unsupported" $(\mathrm{p}<0.05)$; ${ }^{\#}$ : significantly different from chin lift $(\mathrm{p}<0.05)$. Chin lift with CPAP and jaw thrust with CPAP significantly reduced inspiratory time.

had breathing movements that were comparable with healthy, awake children where the phase angle is $<30^{\circ}$ [16]. Мотоуама [3] studied 13 children suffering from clinically significant UAO when a 2 minimum alveolar concentration (MAC: the alveolar concentration of an inhaled anaesthetic that prevents movement in $50 \%$ of patients in response to a standardized stimulus, e.g. surgical incision) of sevoflurane was reached, which was equivalent to $\sim 5 \%$ volume in the present study. In comparison, the present results show that when a lower concentration of sevoflurane (3\% volume) with $50 \%$ oxygen/nitrous oxide is used, it did not necessarily lead to UAO and severe asynchrony of breathing. Although all inhalational anaesthetics decrease muscle tone, sevoflurane has a less deleterious effect on TAA than halothane [17], but the mechanism by which sevoflurane influences TAA is unclear. However, it may be suggested that sevoflurane has a more deleterious effect on patients who already have preexisting UAO (in five of six patients with nocturnal apnoea, phase angle was markedly altered with values $>120^{\circ}$ at baseline). An age-related correlation with the degree of asynchrony was not found in the present study population as has been shown in infants [14]. Breathing patterns remain an indirect assessment of changes in the upper airways, limiting the conclusions drawn in this study. The methods such as imaging of airway changes, flow measurements and analyses of arterial blood gases may further contribute to the utility of the present findings. However, any dynamic imaging with endoscopy may increase upper airway resistance and cause changes in airway geometry. Inductive plethysmography is noninvasive, does not confound observations and is applicable without any ethical reservations.

Chin lift had no effect on, or even worsened TAA. This could be explained by a more severe narrowing of the upper airway when the chin was lifted, which results from an imbalance between inspiratory muscle activity and the negative intraluminal pressure generated during inspiration. Such children are dependent on oral breathing. When the chin lift is performed properly, it cuts off the oral airflow because of the large tonsils. This in turn diverts inspiratory flow to the nasal passages which in this particular case is uniquely shut off by the enlarged adenoids. This explanation is supported by PICKERING and BEARDSMORE [18] who showed that when the chin lift manoeuvre was performed with an almost closed mouth in children with adenotonsillar hypertrophy, there was greater resistance to nasal breathing, caused by the adenoids. However, in a previous study it was demonstrated that in healthy children without adenotonsillar hypertrophy, chin lift is an effective manoeuvre to insure an open airway during propofol anaesthesia [19]. Chin lift widened the airway diameter anterioposteriorly mainly at the area of the epiglottis and transversally at the soft palate [19].

In the children with adenotonsillar hypertrophy, jaw thrust was more effective than chin lift in improving TAA. This was especially seen in patients with severe TAA at baseline and indicated UAO. In these patients, jaw thrust achieved a more synchronous breathing pattern with an improvement in TAA mainly during the inspiratory phase. This indicated that mandibular protrusion, which occurs during jaw thrust, opens the airway more effectively than chin lift. It should be noted, however, that there is no correlation between the degree of the mandibular protrusion and the widening of the pharynx in adults [20]. Unfortunately, no comparable data are available for paediatric patients. Compared with chin lift, jaw thrust has the several theoretical advantages. The tension on the tongue and suprahyoidal muscles is stronger, which pulls the hyoid bone ventrally towards the root of the tongue. This actively widens the pharynx. Furthermore, the mouth is wide open and breathing becomes easier than during chin lift [18].

In a small number of children with adenotonsillar hypertrophy, jaw thrust itself was not sufficient to achieve improvement of TAA: the phase angle in nine of the 27 patients stayed $>90^{\circ}$, whereas five patients with severe TAA completely failed to improve. The mechanisms responsible for these five nonresponders are not clear. Several factors have to be taken into account. First, a pure arytenoid prolapse [21] or another anatomical structure influencing the glottic aperture, e.g. the epiglottis, may lead to a narrowing of the upper airway that will not be cleared effectively by the jaw thrust manoeuvre itself. Secondly, these nonresponders could have had a change in airway resistance without alteration of their breathing pattern. Thirdly, the sevoflurane anaesthesia may also have been causal [3]. With regard to the last possibility, the basis for variable responses of adults or children to sevoflurane anaesthesia is unknown. Finally, the phenomenon did not correlate with age.

By dilating and splinting the upper airway, CPAP can completely eliminate UAO and thus lead to improved breathing patterns [22-24]. The effect of CPAP has been suggested to be multifactorial, including interactions between changes in chest wall stability, pulmonary mechanics, lung volume, and respiratory muscle dynamics $[23,25]$. In the present 
study it is suggested that the increase in thoracoabdominal synchrony during CPAP indicated an improved breathing strategy, and may represent the optimal interaction of all these parameters mentioned above. In severe TAA, both chin lift and jaw thrust in combination with CPAP significantly improved the phase angle and the percentage of time in which the $\mathrm{RC}$ and $\mathrm{AB}$ motion signals were in opposite directions. In five patients with severe TAA, additional CPAP completely failed to improve TAA. However, phase angle cannot be used as an absolute measure of UAO or narrowing because between individuals the distortion of the $\mathrm{RC}$ and, hence, the asynchrony depends on the degree of RC compliance [16]. Moreover, sevoflurane itself can be responsible for TAA without UAO [17].

In conclusion, the results of this study have shown that asynchronous and paradoxical thoraco-abdominal motions often occur in spontaneously breathing children with adenotonsillar hypertrophy during sevoflurane anaesthesia. Chin lift without continuous positive airway pressure should be used with caution in these patients because its efficacy was markedly limited. The reason for this limited effect is presumably because in children with more severe adenotonsillar hypertrophy, chin lift without continuous positive airway pressure converted partial airway obstruction into almost complete airway obstruction. It is this mechanism that leads us to recommend removal of the tonsils and adenoids. In contrast, jaw thrust with or without additional continuous positive airway pressure, especially where there was a severe thoraco-abdominal asynchrony, produced an improvement in pre-existing moderate and severe asynchronous thoraco-abdominal motions.

Acknowledgements. The authors would like to thank J. Etlinger for editorial work.

\section{References}

1. Bluestone CD. Current indications for adenoidectomy. Ann Otol Rhinol Laryngol 1992; 155: S58-S64.

2. Anonymous. Anaesthesia for tonsillectomy. Lancet 1987; $1: 1357-1358$.

3. Motoyama EK. Inspiratory muscle incoordination and upper airway obstruction during inhalation anesthesia. Int Anesthesiol Clin 1997; 35: 45-47.

4. Ahlquvist J, Hultcrantz E, Svanholm H. Children with tonsillar obstruction: indications for and efficacy of tonsillectomy. Acta Paediatr Scand 1988; 77: 831 -835.

5. Hammer J, Newth CJL, Deakers TW. Validation of the phase angle technique as an objective measure of upper airway obstruction. Pediatr Pulmonol 1995; 19: $167-173$.

6. Allen JL, Wolfson MR, McDowell K, Shaffer TH. Thoracoabdominal asynchrony in infants with airflow obstruction. Am Rev Respir Dis 1990; 141: 337-342.

7. Davis GM, Cooper DM, Mitchell I. The measurement of thoraco-abdominal asynchrony in infants with severe laryngotracheobronchitis. Chest 1993; 103: $1842-1848$.

8. Roy WL. Laryngospasm in paediatric anaesthesia. Can J Anaesth 1988; 35: 93 -98.
9. Swan HD, Crawford MW, Pua HL, Stephens D, Lerman J. Additive contribution of nitrous oxide to sevoflurane minimium alveolar concentration for tracheal intubation in children. Anesthesiology 1999; 91: $667-671$.

10. Boidin MP. Airway patency in the unconscious patient. Br J Anaesth 1985; 57: 306-310.

11. Duffty P, Spriet L, Bryan A. Respiratory inductive plethysmography (Respitrace $\mathbb{R})$ : An evaluation of its use in the infant. Am Rev Respir Dis 1981; 123: $542-$ 546.

12. Agostoni E, Mognoni P. Deformation of the chest wall during breathing efforts. J Appl Physiol 1986; 21: $1827-1832$

13. Rusconi F, Gagliardi L, Aston H, Silverman M. Respiratory inductive plethysmography in the evaluation of lower airway obstruction during methacholine challenge in infants. Pediatr Pulmonol 1995; 20: $396-402$.

14. Benameur M, Goldman MD, Ecoffey C, Gaultier C. Ventilation and thoracoabdominal asynchrony during halothane anesthesia in infants. J Appl Physiol 1993; 74: $1591-1596$.

15. Allen JL, Greenspan JS, Deoras KS, Keklikian E, Wolfson MR, Shaffer TH. Interaction between chest wall motion and lung mechanics in normal infants and infants with bronchopulmonary dysplasia. Pediatr Pulmonol 1991; 11: $37-43$.

16. Sivan Y, Deakers TW, Newth CJL. Thoraco-abdominal asynchrony in acute upper airway obstruction in small children. Am Rev Respir Dis 1990; 142: 540-544.

17. Brown K, Aun C, Stocks J, Jackson E, Mackersie A, Hatch D. A comparison of the respiratory effects of sevoflurane and halothane in infants and young children. Anesthesiology 1998; 89: 86-92.

18. Pickering DN, Beardsmore CS. Nasal flow limitation in children. Pediatr Pulmonol 1999; 27: $32-36$.

19. Reber A, Wetzel SG, Schnabel K, Bongatz G, Frei FJ. Effect of combined mouth closure and chin lift on upper airway dimensions during routine magnetic resonance imaging in pediatric patients sedated with propofol. Anesthesiology 1999; 90: 1617-1623.

20. Hochban W, Neumann I, Ziegler A. Mechanisch/ prothetische Unterkieferprotrusion: Auswirkungen auf den Pharynx. [Mechanical/prosthetic protrusion of the mandible: effects on the pharynx]. Pneumologie 1996; 50: $919-923$.

21. Reber A, Paganoni R, Frei FJ. Aiway obstruction due to arytenoid prolapse in a child. Acta Anaesthesiol Scand 1999; 43: 104-106.

22. Waters KA, Everett FM, Bruderer JW, Sullivan CE. Obstructive sleep apnea: the use of nasal CPAP in 80 children. Am J Respir Crit Care Med 1995; 152: 780 785 .

23. Deegan PC, Nolan P, Carey M, McNicholas WT. Effects of positive airway pressure on upper airway dilator muscle activity and ventilatory timing. $J \mathrm{Appl}$ Physiol 1996; 81: 470-479.

24. Burger CD, Stanson AWW, Daniels KK, Sheedy PF, Shepard JW Jr. Fast-CT evaluation of the effect of lung volume on upper airway size and function in normal men. Am Rev Respir Dis 1992; 146: 335-339.

25. Locke R, Greenspan JS, Shaffer TH, Rubenstein SD, Wolfson MR. Effect of nasal CPAP on thoracoabdominal motion in neonates with respiratory insufficiency. Pediatr Pulmonol 1991; 11: 259-264. 